KÖLAI MATH. SEM. REP.

19 (1967), 156-158

\title{
CURVATURE-PRESERVING TRANSFORMATIONS OF K-CONTAC'T RIEMANNIAN MANIFOLDS
}

\author{
By ShûKichi TANNo
}

Let $M$ be a contact Riemannian manifold with a contact form $\eta$, the associated vector field $\xi$, $(1,1)$-tensor field $\phi$ and the associated Riemannian metric $g$. If $\xi$ is a Killing vector field, $M$ is said to be a $K$-contact Riemannian manifold. Further, $M$ is said to be normal, if $\phi$ satisfies the relation

$$
\left(\nabla_{X} \phi\right)(Y)=g(X, Y) \xi-\eta(Y) X
$$

for any vector fields $X$ and $Y$ on $M$, where $\nabla$ is the covariant differentiation with respect to $g$.

Recently Okumura [2] got the following result:

(A) In a normal contact Riemannian manifold, any curvature-preserving infinitesimal transformation is an infinitesimal isometry.

On the other hand, Sakai [3] got the result:

(B) Any affine transformation of a K-contact Riemannian manifold is an isometry.

In this note, we prove the next theorem which covers the above (A) and (B):

Theorem. Let $M, N$ be $K$-contact Riemannian manifolds, then any curvaturepreserving transformation of $M$ to $N$ is an isometry.

The proof of our theorem has similar aspect to that in [3]. In an $m$-dimensional $K$-contact Riemannian manifold we have

$$
\begin{aligned}
& R_{1}(\xi, X)=(m-1) \eta(X), \\
& R(X, \xi) \xi=-X+\eta(X) \xi
\end{aligned}
$$

for any vector field $X$ on $M$, where $R_{1}$ and $R$ denote the Ricci curvature and Riemannian curvature tensor [1].

\section{$\S$ Proof of the theorem.}

We denote the corresponding tensors in $N$ by "'". Let $\varphi$ be a curvaturepreserving transformation of $M$ to $N$ and let $x$ be an arbitrary point of $M$, and we put $y=\varphi x$. By $X, Y, Z, W$ we denote vector fields on $M$. In any Riemannian manifold we have

Received July 7, 1966 . 


$$
\text { ' } g_{y}(' R(\varphi X, \varphi Y) \varphi Z, \varphi W)=-{ }^{\prime} g_{y}(' R(\varphi X, \varphi Y) \varphi W, \varphi Z),
$$

where $\varphi$ stands for the differential of $\varphi$. As $\varphi$ is curvature-preserving: $\varphi(R(X, Y) Z)$ ${ }^{\prime} R(\varphi X, \varphi Y) \varphi Z$, we have

$$
\left(\varphi^{* \prime} g\right)_{x}(R(X, Y) Z, W)=-\left(\varphi^{* \prime} g\right)_{x}(R(X, Y) W, Z) .
$$

If we put $Y=Z=W=\xi$, using (2) we have

$$
\left(\varphi^{* \prime} g\right)_{x}(X, \xi)=\sigma_{x} \eta_{x}(X)
$$

where $\sigma_{x}=\left(\varphi^{* \prime} g\right)_{x}(\xi, \xi)$. Next we put $Y=Z=\xi$, then

$$
\left(\varphi^{* \prime} g\right)_{x}(-X+\eta(X) \xi, W)=-\left(\varphi^{* \prime} g\right)_{x}(R(X, \xi) W, \xi) .
$$

Replace $X$ in (5) by $W$ or $R(X, \xi) W$, then (6) turns to

$$
-\left(\varphi^{* \prime} g\right)_{x}(X, W)+\sigma_{x} \eta_{x}(X) \eta_{x}(W)=-\sigma_{x} \eta_{x}(R(X, \xi) W) .
$$

On the other hand, as

$$
\eta_{x}(R(X, \xi) W)=g_{x}(R(X, \xi) W, \xi)=g_{x}(X, W)-\eta_{x}(X) \eta_{x}(W),
$$

we have $\left(\varphi^{* \prime} g\right)_{x}(X, W)=\sigma_{x} g_{x}(X, W)$. Namely $\varphi$ is a conformal transformation. Next we prove $\sigma_{x}=1$. Put $X=\varphi \xi$ in (1), then we get

$$
{ }^{\prime} R_{1 y}\left({ }^{\prime} \xi, \varphi \xi\right)=(m-1)^{\prime} \eta_{y}(\varphi \xi) \text {. }
$$

Since $\varphi$ also leaves $R_{1}$ invariant, we have

$$
{ }^{\prime} R_{1 y}\left({ }^{\prime} \xi, \varphi \xi\right)=R_{1 x}\left(\varphi^{-1} \prime^{\prime} \xi, \xi\right)=(m-1) \eta_{x}\left(\varphi^{-1}{ }^{\prime} \xi\right) .
$$

From $(7)$ and $(8),{ }^{\prime} \eta_{y}(\varphi \xi)=\eta_{x}\left(\varphi^{-1} \prime \xi\right)$ follows. While we obtain

$$
\begin{aligned}
{ }^{\prime} \eta_{y}(\varphi \xi) & ={ }^{\prime} g_{y}\left({ }^{\prime} \xi, \varphi \xi\right)={ }^{\prime} g_{y}\left(\varphi \cdot \varphi^{-1} \prime \xi, \varphi \xi\right) \\
& =\left(\varphi^{* \prime} g\right)_{x}\left(\varphi^{-1} \prime^{\prime} \xi, \xi\right)=\sigma_{x} g_{x}\left(\varphi^{-1} ' \xi, \xi\right) \\
& =\sigma_{x} \eta_{x}\left(\varphi^{-1} \prime \xi\right) .
\end{aligned}
$$

Thus we get $\sigma_{x}=1$ or ' $\eta_{y}(\varphi \xi)=0$. Suppose that ' $\eta_{y}(\varphi \xi)=0$, then we have ${ }^{\prime} R_{y}\left(\varphi \xi,{ }^{\prime} \xi\right)^{\prime} \xi$ $=-(\varphi \xi)_{y}$ by (2) and so

$$
\begin{aligned}
\sigma_{x} & ={ }^{\prime} g_{y}(\varphi \xi, \varphi \xi) \\
& ={ }^{\prime} g_{y}\left({ }^{\prime} R\left({ }^{\prime} \xi, \varphi \xi\right)^{\prime} \xi, \varphi \xi\right) \\
& ={ }^{\prime} g_{y}\left(\varphi \cdot R\left(\varphi^{-1} \prime^{\prime} \xi, \xi\right) \varphi^{-1} \xi \xi, \varphi \xi\right) \\
& =\left(\varphi^{* \prime} g\right)_{x}\left(R\left(\varphi^{-1} \xi \xi, \xi\right) \varphi^{-1} \xi \xi, \xi\right) \\
& =-\sigma_{x} g_{x}\left(R\left(\varphi^{-1} \prime^{\prime} \xi, \xi\right) \xi, \varphi^{-1} \prime \xi\right) \\
& =\sigma_{x} g_{x}\left(\varphi^{-1} \xi \xi, \varphi^{-1} \xi \xi\right)=1 .
\end{aligned}
$$

Therefore $\sigma$ is equal to 1 on $M$, this completes the proof. 


\section{REFERENCES}

[1] Hatakeyama, Y., Y. Ogawa, and S. Tanno, Some properties of manifolds with contact metric structure. Tôhoku Math. J. 15 (1963), 42-48.

[2] Oкumura, M., Certain infinitesimal transformation of normal contact metric manifold. Kōdai Math. Sem. Rep. 18 (1966), 116-119.

[3] SaKaI, T., Some transformations on $K$-contact and normal contact Riemannian manifolds. Tôhoku Math. J. 18 (1966), 216-224.

Mathematical Institute,

TÔHOKU UNIVERSITY. 